\title{
INSTABILITY OF THE EIKONAL EQUATION AND SHAPE FROM SHADING*
}

\author{
IAN BARNES ${ }^{1}$ AND KEWEI ZHANG ${ }^{1}$
}

\begin{abstract}
In the shape from shading problem of computer vision one attempts to recover the threedimensional shape of an object or landscape from the shading on a single image. Under the assumptions that the surface is dusty, distant, and illuminated only from above, the problem reduces to that of solving the eikonal equation $|D u|=f$ on a domain in $\mathbb{R}^{2}$. Despite various existence and uniqueness theorems for smooth solutions, we show that this problem is unstable, which is catastrophic for general numerical algorithms.
\end{abstract}

Mathematics Subject Classification. 57N05, 57M05, 26A16, 54C20.

Received: 1997. Revised: August 19, 1999.

\section{INTRODUCTION}

Imagine you have a single black-and-white photograph taken from a spacecraft orbiting above the surface of a dusty planet, at an instant when the orbiter and the sun are sufficiently high in the sky that there are no shadows and none of the surface is hidden from view. Can you draw a contour map of the surface with just this information? You have no shadows, no visible edges, no perspective, and no stereoscopic information. In this article we study this problem, the simplest form of the shape from shading problem.

Let us give a brief derivation of how the eikonal equation arises in this context. Suppose the height of a surface above some reference level is given by the function $u=u\left(x_{1}, x_{2}\right)$ defined on some domain $\Omega \subset \mathbb{R}^{2}$. Under the assumption that the viewing point is high above the surface, the image seen will be formed by parallel (or orthographic) projection and will avoid the complications of perspective which arise in close viewing. In this case the brightness of a point on the surface will also be described by a function taking its values on $\Omega$.

In all the analysis that follows, we restrict ourselves to the case where the camera and the light source are vertically above the surface. All our results carry across to the slightly more general situation described above with no fundamental differences, only notational complications.

Suppose further that the light is provided by a distant point source and that the surface is a uniform Lambertian or matte surface. A Lambertian surface is one which appears equally bright from all directions. This is purely diffuse reflection, as opposed to the specular (mirror-type) reflections seen on shiny surfaces. The relative brightness of a small element of a Lambertian surface is determined solely by the amount of light which falls upon it, and in the case of a single overhead light source, this is given by the cosine of the angle between

\footnotetext{
Keywords and phrases. Eikonal equation, shape from shading, instability, numerical analysis.

* This work was supported by the Australian Research Council.

1 Department of Mathematics, Macquarie University, Sydney NSW 2109, Australia.

e-mail: barnes@maths.mq.edu.au; kewei@maths.mq.edu.au
} 
the surface normal vector and the direction of the light source. If we write $D u$ for the gradient of $u$, then the unit normal vector to the graph of $u$ is given by

$$
\vec{n}=\frac{(-D u, 1)}{\sqrt{1+|D u|^{2}}}
$$

and so (ignoring a constant factor depending on the material properties of the surface) the brightness $E\left(x_{1}, x_{2}\right)$ of the point in the image corresponding to the point $\left(x_{1}, x_{2}, u\left(x_{1}, x_{2}\right)\right)$ on the graph of $u$ is given by

$$
E\left(x_{1}, x_{2}\right)=\vec{n} \cdot(0,0,1)=\frac{1}{\sqrt{1+\left|D u\left(x_{1}, x_{2}\right)\right|^{2}}} \cdot
$$

This is Horn's image irradiance equation [10]. If we know the brightness $E$ then we can invert this relation to give

$$
|D u|^{2}=\frac{1}{E^{2}}-1
$$

which is a first-order nonlinear partial differential equation for the unknown height function $u$. Since $0<E \leq 1$, we can set

$$
\mathcal{E}\left(x_{1}, x_{2}\right)=\sqrt{\frac{1}{E\left(x_{1}, x_{2}\right)^{2}}-1}
$$

and obtain the eikonal equation

$$
|D u|=\mathcal{E}
$$

For more general situations and more detailed discussion see [11]. There are several uniqueness results for classical $C^{2}$ solutions of the eikonal equation. See for example $[3,6]$ and Bruss. Lions et al., took a different approach using the notion of viscosity solutions for Hamilton-Jacobi equations [14,17]. They examined existence and uniqueness as well as numerical approximations to viscosity solutions for the eikonal equation and more general equations under various boundary conditions and applied these results to the shape from shading problem.

Horn and Brooks [12] suggested a variational approach to the shape-from-shading problem, in which they sought to minimise the functional

$$
I(u)=\int_{\Omega}|| D u|-\mathcal{E}|
$$

which measures the $L^{1}$ deviation of $|D u|$ from the desired function $\mathcal{E}$. In [4] it was shown that in general this approach is unsatisfactory because there is a certain instability: for any smooth subsolution $v$ of equation (1), there exists a bounded sequence $\left\{u_{n}\right\}$, with $u_{n}=v$ on $\partial \Omega$, and

$$
\int_{\Omega}|| D u_{n}|-\mathcal{E}| \rightarrow 0 \quad \text { and } u_{n} \stackrel{*}{\rightarrow} v \text { in } W^{1, \infty}(\Omega) \text { as } n \rightarrow \infty .
$$

In this paper we improve that result by showing that there exists a sequence $\left\{u_{n}\right\}$ bounded in $W^{1, \infty}(\Omega)$ and agreeing with $v$ on $\partial \Omega$, such that

$$
\left\|\left|D u_{n}\right|-\mathcal{E}\right\|_{L^{\infty}(\Omega)} \rightarrow 0 \quad \text { and } u_{n} \stackrel{*}{\rightarrow} u \text { in } W^{1, \infty}(\Omega) .
$$


In equation (2), an $L^{1}$ norm was used to measure the error in the gradient of the approximate solutions. The disadvantage of that approach is that because the error is measured in an integral average sense it is possible that it can concentrate on a set of small measure. When we view graphs with these types of concentrations, we may see some small bright or dark spots which make the shading of the approximate sequence quite different from that of the graph of a known solution. The measure of error in equation (3) is much stronger since the convergence is almost pointwise, which prevents the formation of spots.

In Section 2 we prove this strengthened instability theorem. In Section 3 we compute some examples numerically and view their graphs in three-dimensional space. We construct exact solutions and members of the sequence of approximate "non-solutions" and show that when viewed under the correct conditions they are indistinguishable, whilst when they are viewed from different directions they are clearly different. We also discuss some of the issues involved in computation, in particular the question of resolution.

\section{INSTABILITY OF THE EIKONAL EQUATION}

We begin by making some standard definitions. Let $\Omega \subset \mathbb{R}^{2}$ be a bounded domain with Lipschitz boundary $\partial \Omega$ and closure $\bar{\Omega}$ and let $C^{k}(\bar{\Omega})$ be the space of restrictions of $k$-times continuously differentiable functions on $\mathbb{R}^{2}$ to $\bar{\Omega}$. We write $C(\bar{\Omega})=C^{0}(\bar{\Omega})$. For $1 \leq p \leq \infty$ let $L^{p}(\Omega)$ be the usual Lebesgue space and $W^{1, p}(\Omega)$ the Sobolev space of functions with weak first derivatives in $L^{p}$. Since $\partial \Omega$ is assumed Lipschitz, elements of $W^{1, p}(\Omega)$ admit traces on $\partial \Omega$ (see [1]) and we can then define

$$
W_{0}^{1, p}(\Omega)=\left\{u \in W^{1, p}(\Omega): \operatorname{trace}(u)=0 \text { on } \partial \Omega\right\} .
$$

If $v: \Omega \rightarrow \mathbb{R}^{2}$ is a vector valued function such that each of its components is in $C^{k}(\bar{\Omega})\left(L^{p}(\Omega)\right.$, respectively), we say that $v$ belongs to $C^{k}\left(\bar{\Omega}, \mathbb{R}^{2}\right)\left(L^{p}\left(\Omega, \mathbb{R}^{2}\right)\right.$, respectively). We assume throughout that $\mathcal{E}=\mathcal{E}(x, y) \in C(\bar{\Omega})$ is non-negative. Weak convergence in $W^{1, p}(\Omega)$ is denoted by $\rightarrow$, weak-* convergence by $\stackrel{*}{\rightarrow}$ and strong convergence by $\rightarrow$. Recall that functions in $W^{1, \infty}(\Omega)$ are locally Lipschitz and hence differentiable almost everywhere (see for example [7]).

We also state a result of real analysis needed in the proof, concerning subdivision of domains into squares. In most applications the domain will be rectangular, in which case subdivision into squares is easy, but this result allows us to consider more general domains. The proof can be found, for example, in reference [18].

Lemma 1. Suppose $\Omega \subset \mathbb{R}^{2}$ is a bounded and open. Then there exists a sequence of open squares $\left\{D_{k}\right\}$, $k=1,2, \ldots$ with side lengths $2^{-h_{k}}$ for some positive integers $h_{k}$, and with their edges parallel to the co-ordinate axes, such that $D_{j} \cap D_{k}=\emptyset$ whenever $j \neq k$ and

$$
\Omega \subseteq \bigcup_{k=1}^{\infty} \overline{D_{k}} \subseteq \bar{\Omega}
$$

The following is our main result.

Theorem 1. Let $\mathcal{E} \in C(\bar{\Omega})$ be a non-negative function and let $v \in C^{1}(\bar{\Omega})$ be a smooth subsolution of the eikonal equation (1) in the sense that

$$
|D v(x)| \leq \mathcal{E}(x)
$$

for all $x \in \Omega$. Then there exists a sequence $\left\{u_{n}\right\} \subset W^{1, \infty}(\Omega)$, satisfying $u_{n}=v$ on $\partial \Omega$, such that

$$
\lim _{j \rightarrow \infty}\left\|\left|D u_{n}\right|-\mathcal{E}\right\|_{L^{\infty}(\Omega)}=0
$$

and $u_{n} \stackrel{*}{\rightarrow} v$ weak-* in $W^{1, \infty}(\Omega)$. 
Let us make a few remarks before giving the proof of the theorem.

1. In fact the approximate sequence $\left\{u_{n}\right\}$ converges uniformly to the subsolution $v$ in $\bar{\Omega}$.

2. Our construction is based on some ideas about Young measures (see, for example $[2,4,19]$ ).

3. The analysis throughout holds for more general reflectance maps. In particular it applies to some models for specular reflections when the viewing and illumination directions are the same.

Proof. Let $u_{n}=v+\phi_{n}$, where $v$ is the smooth subsolution of (1). We construct the sequence $\left\{\phi_{n}\right\} \subset W^{1, \infty}(\Omega)$ such that $\phi_{n} \stackrel{*}{\rightarrow} 0$ in $W^{1, \infty}(\Omega)$ and

$$
\left|D u_{n}\right|=\left|D v+D \phi_{n}\right| \rightarrow \mathcal{E}
$$

in $L^{\infty}(\Omega)$. First we give the construction of the sequence $\phi_{n}$, then prove that it has the desired properties.

Take a decomposition $\mathcal{D}_{1}$ of $\Omega$ into squares as guaranteed by the lemma. The idea is that the graph of $\phi_{1}$ will consist of many small pyramids or tent shapes with these squares as a base. We can then subdivide each of these squares into four to obtain a new decomposition $\mathcal{D}_{2}$ and repeat the construction to obtain $\phi_{2}$ and so on. At the $n$th stage, the side lengths of the squares in the collection $\mathcal{D}_{n}$ are bounded above by $2^{-n}$ (also see $[5,8]$ ).

So now let us consider a single square $D \in \mathcal{D}_{n}$. Suppose its centre is $x^{*}=\left(x_{1}^{*}, x_{2}^{*}\right)$ and its side length is $h$. We construct a continuous and piecewise linear function $\phi: D \rightarrow \mathbb{R}$.

The idea is that the gradient vector $D \phi(x)$ should lie on the circle in $\mathbb{R}^{2}$ centered at $-D v(x)$ with radius $\mathcal{E}(x)$. If we could do this, then we would have $|D v(x)+D \phi(x)|=\mathcal{E}(x)$ on $D$. We approximate this goal by only looking at the value of $D v$ at the central point $x^{*}$. Since $v \in C^{1}$, this becomes a good approximation as the squares get small. In order to construct a simple function whose gradients lie on this circle, we pick the four vectors where the co-ordinate axes intersect the circle. Let

$$
\begin{aligned}
& \alpha_{1}^{+}=-D_{1} v\left(x^{*}\right)+\sqrt{\mathcal{E}\left(x^{*}\right)^{2}-\left(D_{2} v\left(x^{*}\right)\right)^{2}} \\
& \alpha_{1}^{-}=-D_{1} v\left(x^{*}\right)-\sqrt{\mathcal{E}\left(x^{*}\right)^{2}-\left(D_{2} v\left(x^{*}\right)\right)^{2}} \\
& \alpha_{2}^{+}=-D_{2} v\left(x^{*}\right)+\sqrt{\mathcal{E}\left(x^{*}\right)^{2}-\left(D_{1} v\left(x^{*}\right)\right)^{2}} \\
& \alpha_{2}^{-}=-D_{2} v\left(x^{*}\right)-\sqrt{\mathcal{E}\left(x^{*}\right)^{2}-\left(D_{1} v\left(x^{*}\right)\right)^{2}}
\end{aligned}
$$

and then define

$$
\begin{aligned}
& \phi_{1}^{+}(x)=\alpha_{1}^{+}\left(x_{1}-x_{1}^{*}+h / 2\right) \\
& \phi_{1}^{-}(x)=\alpha_{1}^{-}\left(x_{1}-x_{1}^{*}-h / 2\right) \\
& \phi_{2}^{+}(x)=\alpha_{2}^{+}\left(x_{2}-x_{2}^{*}+h / 2\right) \\
& \phi_{2}^{-}(x)=\alpha_{2}^{-}\left(x_{2}-x_{2}^{*}-h / 2\right)
\end{aligned}
$$

for $x \in D$. This only makes sense because $v$ is a subsolution. Now let

$$
\phi(x)=\min \left\{\phi_{1}^{+}(x), \phi_{1}^{-}(x), \phi_{2}^{+}(x), \phi_{2}^{-}(x)\right\}
$$

for $x \in D$. Notice that the graph of $\phi$ is a little tent with four sloping sides. The gradient vectors on the four sides are $\left(\alpha_{1}^{+}, 0\right),\left(\alpha_{1}^{-}, 0\right),\left(\alpha_{2}^{+}, 0\right)$ and $\left(\alpha_{2}^{-}, 0\right)$. So at every point of $D$ (except on the edges of the four triangles, where $\phi$ is not differentiable) we have

$$
\left|D v\left(x^{*}\right)+D \phi(x)\right|=\mathcal{E}\left(x^{*}\right) .
$$

Notice also that if $\left|D v\left(x^{*}\right)\right|=\mathcal{E}\left(x^{*}\right)$, this construction gives $\phi(x)=0$ for all $x \in D$. 
We build up the function $\phi_{n}$ on all of $\Omega$ by adding together the pieces supported on each square constructed as above. It is immediate from this construction that $\phi_{n} \in W_{0}^{1, \infty}(\Omega)$. We seek to prove that

1. $\phi_{n} \stackrel{*}{\rightarrow} 0$ as $n \rightarrow \infty$;

2. ||$\left|D v+D \phi_{n}\right|-\mathcal{E} \|_{L^{\infty}(\Omega)} \rightarrow 0$ as $n \rightarrow \infty$.

To prove the first assertion, we first show that $\phi_{n} \rightarrow 0$ in $L^{\infty}(\Omega)$. Now

$$
\begin{aligned}
\left\|\phi_{n}\right\|_{L^{\infty}(\Omega)} & =\sup _{D \in \mathcal{D}_{n}}\left\|\phi_{k}\right\|_{L^{\infty}(D)} \\
& \leq \sup _{D \in \mathcal{D}_{n}} h \mathcal{E}\left(x^{*}\right) \\
& \leq\|\mathcal{E}\|_{C(\bar{\Omega})} \sup _{D \in \mathcal{D}_{n}} h \\
& \leq 2^{-n}\|\mathcal{E}\|_{C(\bar{\Omega})} \rightarrow 0 \quad \text { as } n \rightarrow \infty .
\end{aligned}
$$

Next we show that $D \phi_{n} \stackrel{*}{\rightarrow} 0$ in $L^{\infty}\left(\Omega, \mathbb{R}^{2}\right)$. Let $g \in L^{1}\left(\Omega, \mathbb{R}^{2}\right)$ be fixed and choose any $\epsilon>0$. Since $C_{0}^{1}\left(\Omega, \mathbb{R}^{2}\right)$ is dense in $L^{1}\left(\Omega, \mathbb{R}^{2}\right)$, there exists some function $g_{\epsilon} \in C_{0}^{1}\left(\Omega, \mathbb{R}^{2}\right)$ such that $\left\|g-g_{\epsilon}\right\|_{L^{1}(\Omega)}<\epsilon$. We then have

$$
\left|\int_{\Omega} g \cdot D \phi_{n}\right| \leq\left|\int_{\Omega}\left(g-g_{\epsilon}\right) \cdot D \phi_{n}\right|+\left|\int_{\Omega} g_{\epsilon} \cdot D \phi_{n}\right|
$$

For the first term

$$
\begin{aligned}
\left|\int_{\Omega}\left(g-g_{\epsilon}\right) \cdot D \phi_{n}\right| & \leq\left\|D \phi_{n}\right\|_{L^{\infty}(\Omega)}\left\|g-g_{\epsilon}\right\|_{L^{1}(\Omega)} \\
& \leq \epsilon\left\|D \phi_{n}\right\|_{L^{\infty}(\Omega)} \\
& \leq \epsilon\|\mathcal{E}\|_{C(\bar{\Omega})}
\end{aligned}
$$

In the second term, since $g_{\epsilon} \in C_{0}^{1}(\Omega)$ we can integrate by parts

$$
\begin{aligned}
\left|\int_{\Omega} g_{\epsilon} \cdot D \phi_{n}\right| & =\left|\int_{\Omega} \phi_{n} \operatorname{div} g_{\epsilon}\right| \\
& \leq\left\|\phi_{n}\right\|_{L^{\infty}(\Omega)}\left\|\operatorname{div} g_{\epsilon}\right\|_{C(\Omega)}
\end{aligned}
$$

Since $g_{\epsilon}$ is fixed and $\phi_{n} \rightarrow 0$ in $L^{\infty}$, there is a number $N$ such that $n \geq N$ implies $\left\|\phi_{n}\right\|_{L^{\infty}(\Omega)}<\epsilon /\left\|\operatorname{div} g_{\epsilon}\right\|_{C(\Omega)}$, and so for all $n \geq N$ we have

$$
\left|\int_{\Omega} g \cdot D \phi_{n}\right| \leq \epsilon\left(1+\|\mathcal{E}\|_{C(\bar{\Omega})}\right)
$$

and so $\phi_{n} \stackrel{*}{\rightarrow} 0$ in $W^{1, \infty}(\Omega)$ as $n \rightarrow \infty$.

We now seek to prove the second assertion, that $\left|D u_{n}\right| \rightarrow \mathcal{E}$ in $L^{\infty}$. First consider a single square $D_{k}$ in the decomposition for $\phi_{n}$. Since $\phi_{n}(x)$ is the minimum of the four affine functions $\phi_{n}^{j}(x)$, we can just consider one 
of these at a time. We have

$$
\begin{aligned}
\left\|\left|D u_{n}\right|-\mathcal{E}\right\|_{L^{\infty}\left(D_{k}\right)} & \left\|\left|D v+D \phi_{n}\right|-\mathcal{E}\right\|_{L^{\infty}\left(D_{k}\right)} \\
\leq & \left\|\left|D v+D \phi_{n}^{j}\right|-\left|D v\left(x^{*}\right)+D \phi_{n}^{j}\right|\right\|_{L^{\infty}\left(D_{k}\right)}+\left\|\left|D v\left(x^{*}\right)+D \phi_{n}^{j}\right|-\mathcal{E}\left(x^{*}\right)\right\|_{L^{\infty}\left(D_{k}\right)} \\
& +\left\|\mathcal{E}\left(x^{*}\right)-\mathcal{E}\right\|_{L^{\infty}\left(D_{k}\right)} \\
= & A+B+C .
\end{aligned}
$$

Here $D \phi$ refers to the function $x \mapsto D \phi(x)$ while $D \phi\left(x^{*}\right)$ means the constant function with that value.

Now the triangle inequality gives

$$
A \leq\left\|D v-D v\left(x^{*}\right)\right\|_{L^{\infty}\left(D_{k}\right)}
$$

Since $v \in C^{1}(\bar{\Omega})$, the gradient $D v$ is uniformly continuous and so as $n \rightarrow \infty$ and the squares $D_{k}$ get small, we have $A \rightarrow 0$ (uniformly for all the squares).

The second term $B$ is always zero by the construction of the functions $\phi_{n}^{j}$, and the third term behaves like the first, since we assumed $\mathcal{E}$ was uniformly continuous in $\bar{\Omega}$. So we have

$$
\left\|\left|D u_{n}\right|-\mathcal{E}\right\|_{L^{\infty}(\Omega)} \rightarrow 0 \quad \text { as } n \rightarrow \infty
$$

which completes the proof.

Corollary 1. Let $\mathcal{E}(x) \in C(\bar{\Omega})$ be a non-negative function. Then there exists a sequence $\left\{u_{n}\right\}$ in $W^{1, \infty}(\Omega)$ with $\left.u_{n}\right|_{\partial \Omega}=0$, such that

$$
\lim _{n \rightarrow \infty}\left\|\left|D u_{n}\right|-\mathcal{E}\right\|_{L^{\infty}(\Omega)}=0
$$

and $u_{n} \stackrel{*}{\rightarrow} 0$ weak-* in $W^{1, \infty}(\Omega)$ as $n \rightarrow \infty$.

Proof. The corollary is a direct consequence of the Theorem because $v \equiv 0$ in $\bar{\Omega}$ is always a subsolution of equation (1).

\section{NumERICAL EXPERIMENTS}

We have performed numerical experiments which illustrate the application of this theorem. In all cases we start with a height function $u$ and use the reflectance map to calculate the brightness function $\mathcal{E}$ so that $u$ is automatically a classical solution to $|D u|=\mathcal{E}$. Then starting with a subsolution $v$, we calculate members of the approximating sequence $\left\{u_{n}\right\}$ so that $u_{n} \stackrel{*}{\rightarrow} v$ in $W^{1, \infty}$ (and in particular uniformly) but $\left|D u_{n}\right| \rightarrow \mathcal{E}$ uniformly.

Some parts of this process were carried out using publicly available software packages, while for other steps we wrote simple C programs. Three-dimensional visualisation was done on screen using Geomview [13, 16]. Some of the final images were produced using the Blue Moon Rendering Tools ray-tracing software [9], which implements the RenderMan [15] standard for 3D scene description.

In Figures 1 and 2 the starting function was $u\left(x_{1}, x_{2}\right)=r \sin (2 \pi r)$ where $r=\sqrt{x_{1}^{2}+x_{2}^{2}}$ and the subsolution was the zero function. In Figure 1 we show an approximating function $u_{n}$ corresponding to decomposition of the unit square into $16 \times 16$ and in Figure 2, $40 \times 40$ small squares. 

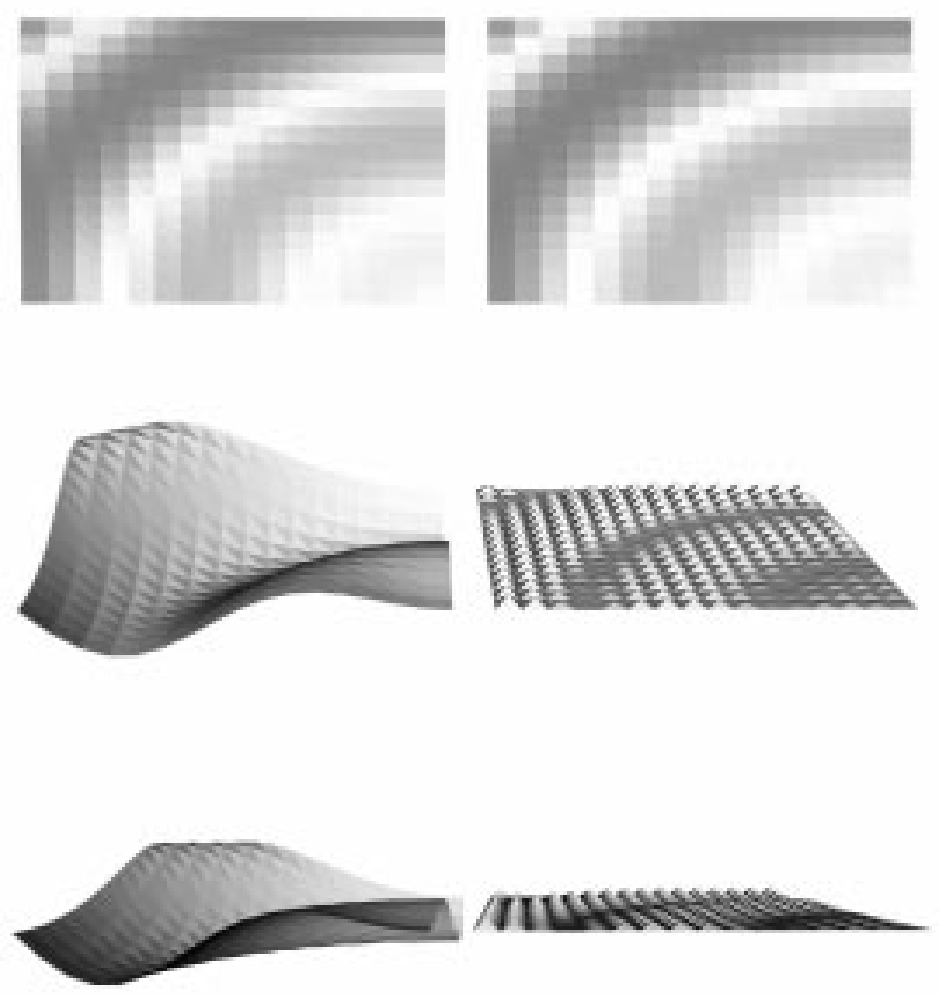

FiguRE 1. Illustration of the instability in the shape-from-shading problem. The images show the view from different directions, of two different landscapes. The landscape on the left is a triangulation of the surface $z=r \sin (2 \pi r)$ where $r=\sqrt{x^{2}+y^{2}}$ on the unit square $0 \leq x \leq 1$, $0 \leq y \leq 1$. Think of this as the graph of an exact solution $u$ to the eikonal equation. The landscape on the right is the graph of one of the approximate solutions $u_{k}$ constructed in the proof of the theorem, with the subsolution $v$ identically zero. The size of the pyramids has been chosen to match the triangulation used on the left. The resolution is $16 \times 16$. In the top pair the scene is viewed from directly above.
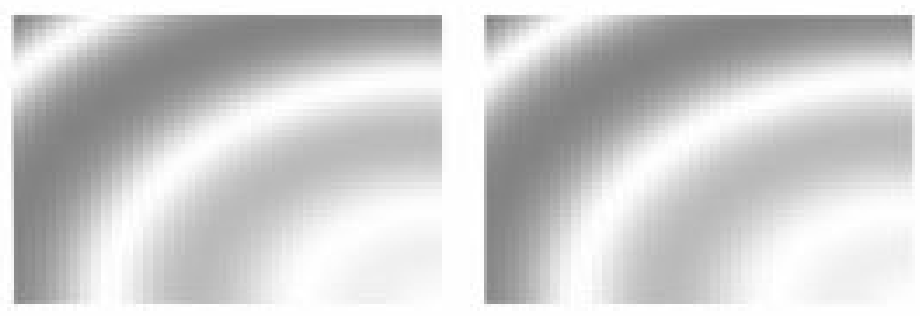

Figure 2. The same as the top pair of images in Figure 1, except that the resolution has been increased to $40 \times 40$. 

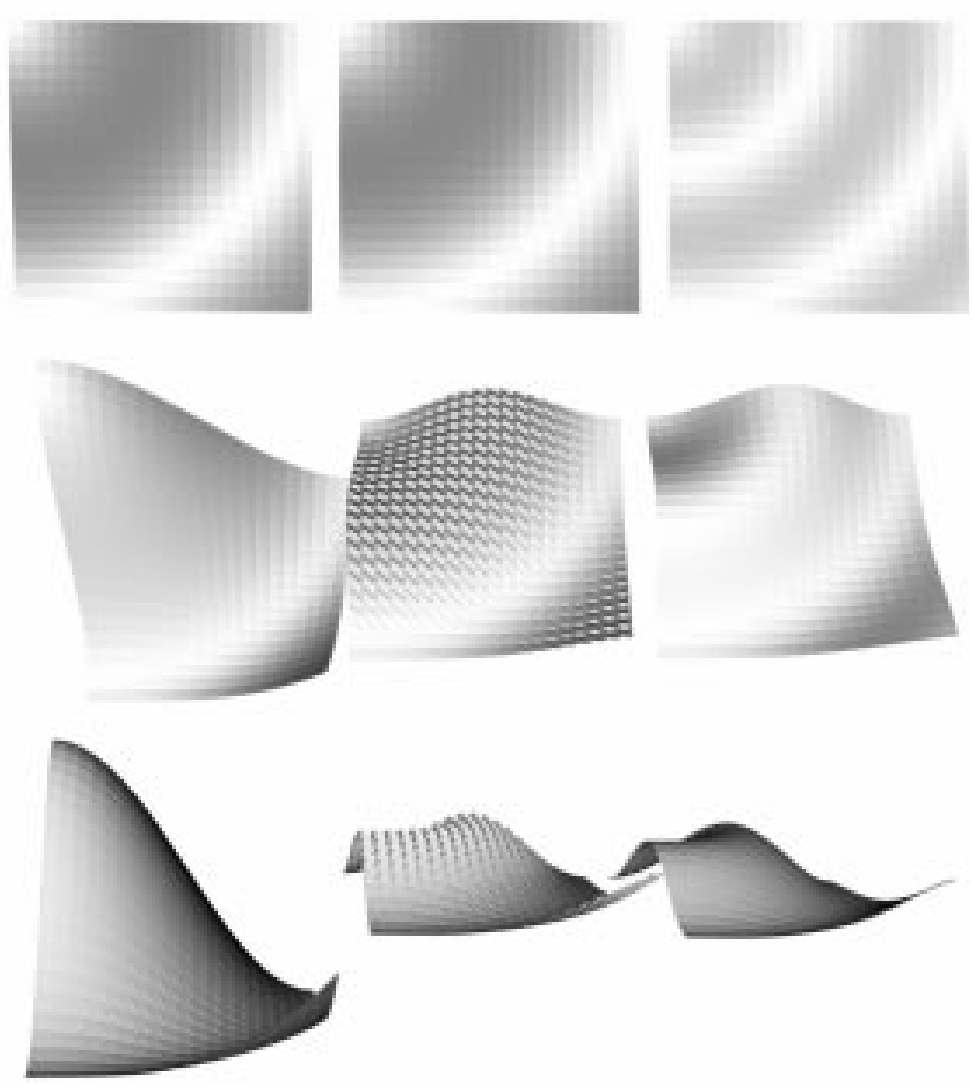

FIGURE 3. Illustration of the case in which the subsolution $v$ is not the zero function. The figure shows three surfaces viewed from three different directions. In the top row, the surfaces are viewed from far away and directly overhead. In each row the left-hand landscape is a triangulation of the graph of the exact solution $u$, which is here given by the equation $z=$ $\cos (\pi r)$, and the right-hand landscape is a triangulation of the surface $z=\frac{1}{4}(1-\cos (2 \pi r))$, which is the graph of a subsolution $v$. The central landscape is the graph of an approximate solution $u_{n}$ as constructed in the proof of the theorem (but modified as discussed in the text so that the pyramids have triangular rather than square bases). It has small pyramids with bases on the right-hand surface but gradient everywhere matching that of the left-hand surface. The resolution is $20 \times 20$.

Figure 3 shows the case in which the subsolution $v$ is not the zero function. Here the starting function is $u\left(x_{1}, x_{2}\right)=\cos (\pi r)$ and the subsolution is $v\left(x_{1}, x_{2}\right)=\frac{1}{4}(1-\cos (2 \pi r))$. The pyramids added to this surface hide the more rapid oscillation of $v$ when seen from the correct direction. Figure 4 shows a close-up side view of these surfaces, showing how the smooth surfaces are triangulated on the same scale as the rough one.

As is clear in all the figures, we approximate the graphs of the "true" solutions by triangulation, interpolating small triangular elements between neighbouring function values. In practice and especially to simplify the construction in the case of a non-zero subsolution, we made a minor modification to the procedure given in the proof, constructing pyramids with triangular rather than square bases. This amounts to building little irregular tetrahedral additions which fit over the triangular facets in the graph of the "true" solution $u$.

This point is worth a little explanation. In applications we generally do not know a function other than as an array of its values. This is particularly true in image processing where our photograph will be available as 


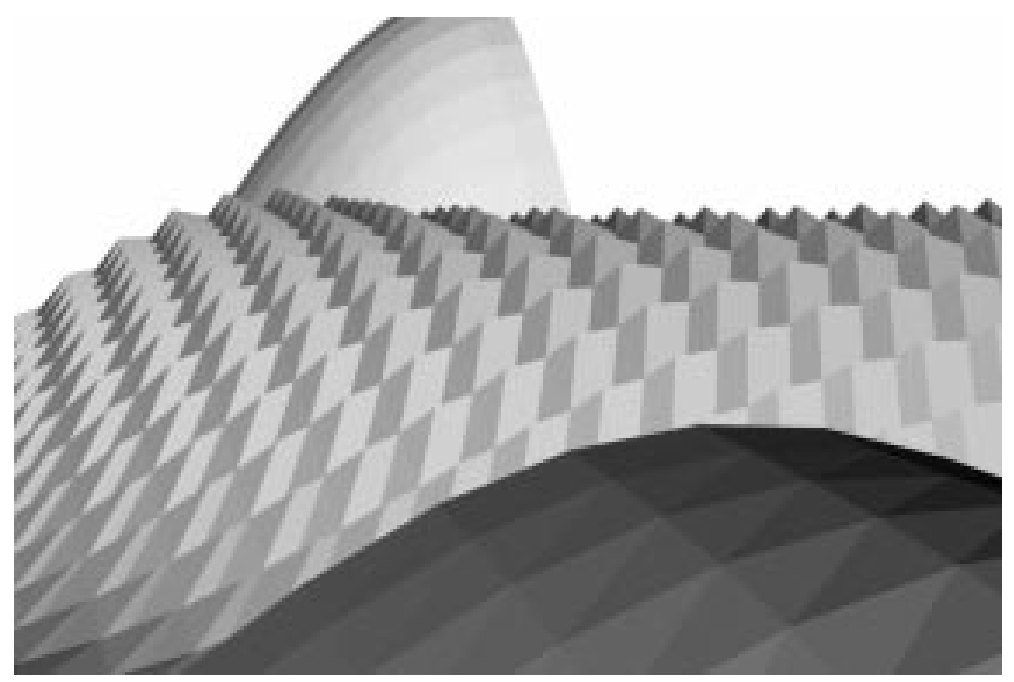

Figure 4. A close-up view of the landscapes depicted in Figure 3. In the distance we see part of the triangulated graph of the exact solution $u$, in the middle distance part of the approximate solution $u_{n}$ and in the foreground part of the triangulated graph of the subsolution $v$.

an array of greyscale brightness values (see Fig. 5). In our illustrations here we have deliberately used extremely coarse grids to make our procedure clear, but there is no impediment to creating one pyramid per image pixel. As long as the pyramid sizes match the grid size of the original data, the resulting images are identical, rather than merely similar. It should be noted that this means using two or three times as many triangles as in the triangulation of the "smooth" surface.

The main point of this is to make it clear that there is no hope of a general shape from shading algorithm; there is simply not enough information in a single image to allow unambiguous reconstruction, even in ideal circumstances. This result should not be too surprising. All is not lost however. Effective algorithms are certainly possible, but they must make assumptions about the nature of the objects being reconstructed. The finite resolution of real image data makes an abstract smoothness assumption (that solutions must be $C^{2}$ for example) irrelevant. What is needed instead is some assumption about the scale on which objects are smooth or rough, and what sort of smoothness or roughness is present. Alternatively perhaps it is possible to formulate some sort of assumption that the view we have of an object is in some way generic: that if we reconstruct the object, and then make a small change in the viewing angle, there should be only a small difference in the computed images.

\section{Appendix}

In this appendix we give a strengthened version of our main result provided us by the referee, Professor Bernard Dacorogna. We thank Professor Dacorogna for allowing us to include this in our article.

Theorem 2. (Dacorogna) Let $\mathcal{E} \in C(\bar{\Omega}), \mathcal{E} \geq 0$ and $v \in C^{1}(\bar{\Omega})$ be such that

$$
|D v(x)| \leq \mathcal{E}(x) \quad \text { for all } x \in \Omega .
$$

Then there exists $u_{j} \in W^{1, \infty}(\Omega)$ such that

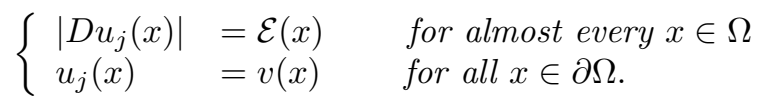

Furthermore $u_{j} \stackrel{*}{\rightarrow} v$ weak-* in $W^{1, \infty}(\Omega)$. 

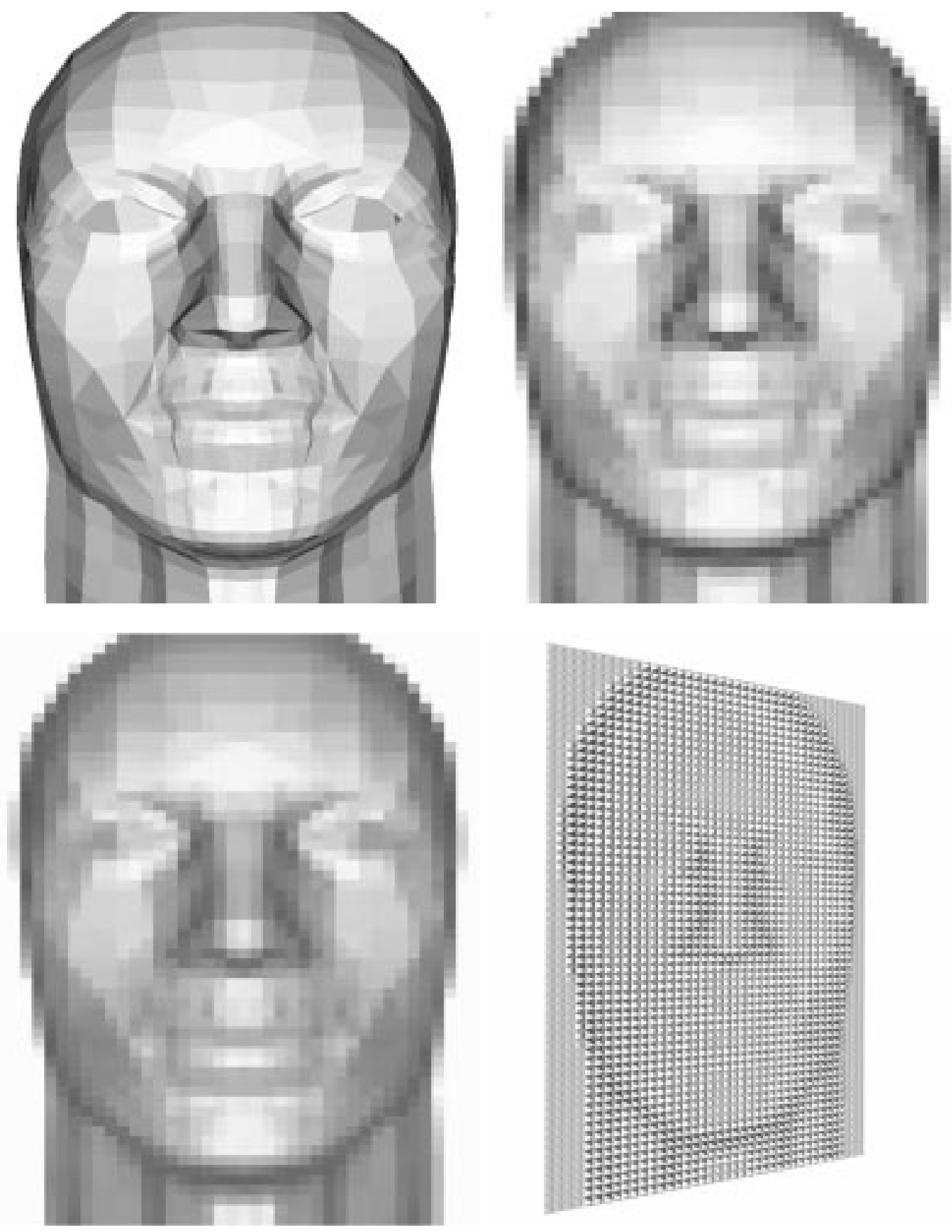

Figure 5. The image of a human head at top left was produced from three-dimensional data, under the usual assumptions that the surface is Lambertian and that the light source and camera are at infinity in the same direction. At top right the same image has been "pixelised" to reduce the resolution to $60 \times 40$. Such an image might result if one had a very poor camera, for example. The lower images show the results of imitating this three-dimensional shape with many little pyramids on a flat background. The image at bottom left is viewed from head-on and is almost indistinguishable from that at top-right. 
Proof. Take $B \subset \mathbb{R}^{n}$ to be the unit ball (or any other regular set) and for every $j \in \mathbb{N}$ make a Vitali covering of $\Omega$ (see for example [18]) by dilations and translations $B_{k}^{j}$ of $B, k=1, \ldots \infty$, all of them with measure less than $1 / j$ such that

$$
\bar{\Omega}=\left(\bigcup_{k=0}^{\infty} \bar{B}_{k}^{j}\right) \cup N^{j}
$$

where $\mu\left(N^{j}\right)=0$ and $\mu\left(B_{k}^{j}\right) \leq 1 / j$ for each $k$. Here $\mu$ refers to Lebesgue measure on $\mathbb{R}^{2}$.

Then solve (by any method, in particular, there is an explicit viscosity solution [14,17])

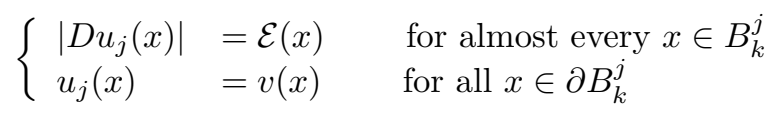

on each of the balls $B_{k}^{j}$. Letting $u_{j}=v$ on $N^{j}$, we have thus defined $u_{j}$ with all claimed properties.

Remark A1 (Dacorogna). This theorem can be further improved a great deal in the following directions.

1. The boundary data $v$ can be chosen in $W^{1, \infty}(\Omega)$ instead of $C^{1}(\bar{\Omega})$.

2. The result is still valid if $H: \mathbb{R}^{n} \rightarrow \mathbb{R}$ is convex and the compatibility condition is

$$
H(D v(x)) \leq \mathcal{E}(x) \quad \text { for all } x \in \Omega .
$$

3. Even when $H$ is non-convex (or even when the problem is vectorial) the same result holds provided the following problem has a $W^{1, \infty}$ solution

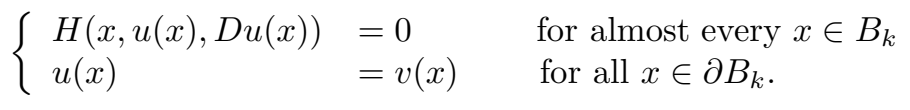

Of course when $H$ is convex and the equation is of the form $H(D u(x))-E(x)=0$ then (6) guarantees (it is even a necessary and sufficient condition) the existence of a Lipschitz solution of (7) (for example the viscosity solution).

Remark A2 (Barnes and Zhang). The above result is certainly stronger and more general than ours. It also shows that the eikonal equation (and other related equations) can have infinitely many solutions. We want to make the point however, that our approach is directly related to the numerical analysis. Our proof is essentially our numerical scheme.

Dacorogna's method is not suitable for developing numerical schemes. If we use a Vitali covering by balls, then at every step we need infinitely many balls. If we use any finite number of balls, there will be many interior regions which cannot be covered. This will create visible spots in the images of the approximate solutions.

Suppose instead that we use squares to cover the domain and calculate viscosity solutions on each square. Then on each square we have to find a numerical approximation to the viscosity solution. It is known that the viscosity solution is unique only under very delicate condtions on $\mathcal{E}(x)[14,17]$. So we face difficulties of deciding which solution to choose on each square. Even having managed that, the task of computing an approximate solution on each square is much more complicated than that of calculating a pyramid or tent function on the square.

We believe the two approaches complement each other - the more abstract method proves a stronger version of the result, while the more concrete method provides interesting numerical applications. 


\section{REFERENCES}

[1] R.A. Adams, Sobolev Space. Academic Press New York (1975).

[2] J.M. Ball, A version of the fundamental theorem for Young measure. Lect. Notes Phys. Springer Verlag 344 (1988) $207-215$.

[3] A.R. Bruss, results applicable to computer vision. J. Math. Phys. 23 (1982) 890-896.

[4] J. Chabrowski and K.-W. Zhang, On shape from shading problem Functional Analysis, Approximation Theory and Numerical Analysis, J.M. Rassias Ed., World Scientific (1994) 93-105.

[5] B. Dacorogna Direct Methods in the Calculus of Variations. Springer-Verlag (1989).

[6] P. Deift and J. Sylvester, Some remarks on the shape-from-shading problem in computer vision. J. Math. Anal. Appl. 84 (1981) $235-248$.

[7] L.C. Evans and R.F. Gariepy, Measure Theory and Fine Properties of Functions. Stud. in Adv. Math. CRC Press, Boca Raton (1992).

[8] I. Ekeland and R. Temam, Analyse convexe et problèmes variationnels. Dunod Paris (1974).

[9] L. Gritz, Blue Moon Rendering Tools: Ray tracing software available from ftp://ftp.gwu.edu/pub/graphics/BMRT (1995).

[10] B.K.P. Horn, Robot Vision. Engineering and Computer Science Series, MIT Press, MacGraw Hill (1986).

[11] B.K.P. Horn and M.J. Brooks, Shape from Shading. Ed. MIT Press Ser. in Artificial Intelligence (1989).

[12] B.K.P. Horn and M.J. Brooks, Variational Approach to Shape from Shading in [11]

[13] S. Levy, T. Munzner and M. Phillips, Geomview Visualisation software available from ftp.geom.umn.edu or http://www.geom.umn.edu/locate/geomview

[14] P.-L. Lions, E. Rouy and A. Tourin, Shape-from-shading, viscosity solutions and edges. Numer. Math. 64 (1993) $323-353$.

[15] Pixar, The RenderMan Interface, version 3.1, official specification. Pixar (1989)

[16] M. Phillips, S. Levy and T. Munzner, Geomview: An Interactive Geometry Viewer. Notices Amer. Math. Soc. 40 (1993) 985-988.

[17] E. Rouy and A. Tourin, A viscosity solution approach to shape-from-shading. SIAM J. Numer. Anal. 29 (1992) 867-884.

[18] E.M. Stein, Singular Integrals and Differentiability Properties of Functions. Princeton University Press (1970).

[19] L. Tartar, Compensated compactness and partial differential equations, in Microstructure and Phase Transitions, D. Kinderlehrer et al. Eds., Springer Verlag (1992). 